Canadian

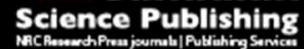

Canadian Journal of Chemistry Revue canadienne de chimie

\title{
Submicron Probes for Scanning Electrochemical Microscopy
}

\begin{tabular}{|r|l|}
\hline Journal: & Canadian Journal of Chemistry \\
\hline Manuscript ID & cjc-2017-0611.R1 \\
\hline Manuscript Type: & Article \\
\hline Date Submitted by the Author: & 22-Nov-2017 \\
\hline $\begin{array}{r}\text { Complete List of Authors: } \\
\text { Is the invited manuscript for } \\
\text { consideration in a Special } \\
\text { Issue?: }\end{array}$ & $\begin{array}{l}\text { Li, Michelle; The University of Western Ontario } \\
\text { Ding, Zhifeng; The University of Western Ontario, }\end{array}$ \\
\hline Keyword: & $\begin{array}{l}\text { Scanning electrochemical microscopy, SECM imaging, laser-pulled Pt } \\
\text { probes, independently addressable microband electrodes, finite element } \\
\text { analysis }\end{array}$ \\
\hline \multicolumn{2}{|l}{} \\
\hline
\end{tabular}


5 Department of Chemistry, The University of Western Ontario, 1151 Richmond Street, London, 


\title{
Submicron Probes for Scanning Electrochemical Microscopy
}

\author{
Michelle S. M. Li, Fraser P. Filice, and Zhifeng Ding*
}

\section{Abstract}

13 To improve the spatial resolutions of scanning electrochemical microscopy (SECM) imaging, the

14 laser-pulled submicron electrode fabrication method was explored in this work. Manual

15 polishing of a laser-pulled Pt nanoelectrode exposed a Pt tip diameter of $250 \mathrm{~nm}$ with a ratio of

16 the tip glass to exposed Pt disc (RG) 30. This fabricated submicron probe was then utilized to

17 study the electrochemical functionality of an independently addressable microband electrode

18 (IAME) sample using SECM. In the constant imaging mode of SECM, where the probe is

19 scanned linearly across the sample at a fixed $z$ position, SECM demonstrated higher resolution

20 than that of the conventional micrometer electrodes when the feedback currents from the Pt and

21 glass microbands were characterized. In addition, the depth scan imaging mode of SECM was

22 also used to extract experimental horizontal line scans and probe approach curves for analysis.

23 3D simulations of the IAME-SECM probe experiments were explored for the first time to

24 quantify the tip-to-sample distances, tilt angle of the sample (or electrode) and height of the Pt

25 microbands. The experimentally characterized height was found to be similar to manufacturer

26 specification (125 nm vs $110 \mathrm{~nm})$. Furthermore, the more computationally-demanding 3D

27 simulation of the true IAME sample geometry (110 $\mathrm{nm}$ height of the Pt microbands) revealed

28 minimal difference in feedback behaviors in comparison to the idealized flat geometry. The

29 removal of this simulation complexity was proved to be sufficient for SECM analysis of the

30 IAME sample by a $250 \mathrm{~nm}$ Pt probe, which saves greatly the computation resources. 
31 Keywords: Scanning electrochemical microscopy, SECM imaging, laser-pulled Pt probes,

32 independently addressable microband electrodes, finite element analysis 


\section{Introduction}

Since its emergence in the late $1980 \mathrm{~s},{ }^{1-4}$ scanning electrochemical microscopy (SECM)

37 has been used for a variety of applications, including but not limited, to microstructure

38 fabrication, ${ }^{5-6}$ probing sample kinetics, ${ }^{7-8}$ and electrochemical imaging. ${ }^{9-11,12,13}$ This technique

39 utilizes a biased electrode, less than $25 \mu \mathrm{m}$ in diameter, that is scanned with extreme precision

40 over a sample submerged in an electrolyte solution..$^{11,14-17}$ Due to the potential bias and probe

41 size, the redox mediator is fully converted to its oxidized or reduced form at the electrode tip in a

42 diffusion-controlled process. When the biased probe is in close proximity to a sample (within

43 distances of several tip radii away), the feedback current will deviate from the steady state

44 current since the flux of the mediator from bulk solution to the tip is no longer a diffusion-

45 controlled process. If the sample is conductive, the tip current will increase since cyclic

46 regeneration of the mediator at the sample-electrolyte interface (positive feedback) increases as

47 tip-to-sample distance is minimized. However, if the sample is insulating, the current will

48 decrease due to the depletion of the mediator at the electrode tip (diffusion-limited, negative

49 feedback).

This relationship between tip current and probe position can be represented as single line

52 scans or in the form of multiaxis current maps (images). In the Ding lab, SECM depth scan

53 imaging is often used since the tip current and probe coordinates $(x, z)$ are embedded into a 2D

54 image. ${ }^{18-20}$ A significant advantage to this method is that each image provides hundreds of

55 usable experimental horizontal line scans and probe approach curves. In addition, this method

56 also provides the real-time generation of the SECM image which allows the experimentalist to 
57 monitor the feedback current and electrode's position relative to the sample. This may prevent 58 collision damage between the SECM tip and the sample.

Resolution of the SECM instrument is commonly limited by electrode size. Larger

61 SECM probes provide stronger electrochemical feedback, however, that feedback is comprised

62 of an averaged signal over the entire electrode surface area. By reducing the electrode size,

63 smaller sample features or regions of increased electrochemical activity can be observed. Here,

64 we use the laser-pulled nanoelectrode fabrication method ${ }^{21-23}$ to greatly reduce the commercially

65 obtainable Pt wire from $25 \mu \mathrm{m}$ diameter to the submicron (nanometer) scale. This reduction in

66 Pt diameter would greatly improve the resolution of SECM images relative to micrometer

67 electrodes. To demonstrate the improvement in SECM resolution, an independently addressable

68 microband electrodes (IAME) sample was characterized by the use of a manually polished laser-

69 pulled $250 \mathrm{~nm}$ Pt electrode.

3D finite elemental analysis simulations of the depth scan experiment were carried out

72 for the first time to fit to horizontal and vertical line scans extracted from the experimental

73 SECM depth scan image, which allows quantitative analysis of sample characteristics, such as

74 the physical properties and reaction kinetics. ${ }^{24}$ These simulations provide insight into the effects

75 of tilt angle, microband height and feedback current in SECM when a probe significantly smaller

76 in stature, relative to the microbands of the IAME ( $5 \mu \mathrm{m}$ band width), is used. Furthermore, a

77 comparison between an idealized IAME sample geometry (where Pt microband heights are

78 ignored) and a computationally-demanding 3D simulation of the true IAME sample geometry

79 was explored. These results indicate the extended complexity of the simulation geometry (and 
80 therefore higher utilization of computational resources) may not be required for SECM analysis

81 of the IAME sample by a $250 \mathrm{~nm}$ Pt probe.

82

\section{Experimental}

\section{$84 \quad$ Materials}

Hexaamineruthenium(III) chloride $\left(\mathrm{Ru}\left(\mathrm{NH}_{3}\right)_{6} \cdot \mathrm{Cl}_{3}, 98 \%\right)$ and potassium chloride $(\mathrm{KCl}$,

86 99\%) were acquired from Sigma-Aldrich (Mississauga, ON) and Alfa Aesar (Ward Hill, MA),

87 respectively. $10 \mathrm{mM} \mathrm{Ru}\left(\mathrm{NH}_{3}\right)_{6}{ }^{3+}$ with $0.1 \mathrm{M} \mathrm{KCl}$ as the supporting electrolyte was prepared in

88 deionized water (18 M $\Omega . c m$, MilliQ water, Etobicoke, ON). The Pt IAME sample was

89 purchased from ABTECH Scientific, Inc. (IAME 0504-Pt, Richmond, VA). This IAME has 4

90 alternating (independently addressable) Pt and glass microbands, each $5 \mu \mathrm{m}$ in width. The Pt

91 microbands are $110 \mathrm{~nm}$ in height according to manufacturer specifications (100 $\AA$ TilW and

$921000 \AA \mathrm{Pt})$.

\section{Electrode Fabrication}

94 The nanoelectrodes were fabricated in-house using a P-2000 laser-based micropipette

95 puller (Sutter Instrument, Novato, CA) adapted from the established procedure reported by the 96 Mauzeroll Group. ${ }^{21,25-26}$ Briefly, a $25 \mu \mathrm{m}$ Pt wire (Goodfellow Metals, Cambridge, UK) was

97 inserted into a quartz glass capillary tubes (o.d.: $1.00 \mathrm{~mm}$, i.d.: $0.50 \mathrm{~mm}$, length: $10.00 \mathrm{~cm}$, Sutter

98 Instrument). In place of a single line setting used by the Mauzeroll Group, two single line

99 settings on the P-2000 puller were required in our experimental configuration. A custom-

100 designed vacuum line was applied to the two ends of the micropipette and Pt wire set to be heat- 
101 sealed and pulled. The first of the two is a partial quartz seal program using the line setting of 102 Heat: 580, Filament: 003, Velocity: 140, Delay: 060, Pull: 000. This was followed by the 103 complete quartz seal at Heat: 580, Filament: 002, Velocity: 140, Delay: 060, Pull: 000. These 104 two quartz seal programs were performed with the linear carriages locked in place, securing the 105 capillary in a fixed position. These two line settings were found to improve the integrity of the 106 Pt wire for our experimental configuration. Once the Pt was completely sealed within the quartz 107 capillary, the locking of the linear carriages were removed, followed by the final pull step at a 108 single line setting of Heat: 780, Filament: 002, Velocity: 060, Delay: 140, Pull: 200 was 109 employed. This fabrication procedure produced two Pt nanoelectrodes (NEs). Freshly pulled Pt NEs were first checked optically under a microscope for obvious 111 breakages in Pt wire, followed by extension of the internal electrical connections. Scanning 112 electron microscopy (SEM) was used to confirm the reduction in Pt tip size and RG values (Fig.

113 1). Typically, SEM revealed that the Pt wires were recessed within the pulled quartz capillary

114 (Fig. 1a). Focused ion beam (FIB)-milling and SEM imaging were performed using a LEO 115 Zeiss $1540 \mathrm{XB}$ (Zeiss, Oberkochen, Germany). Up to a thousand times reduction in Pt diameter 116 size, from $25 \mu \mathrm{m}$ to $25 \mathrm{~nm}$, as well as RG values greater than 25 were observed (Fig. 1b). In 117 addition to FIB-milling, the Pt tips were exposed using a homemade polishing wheel attached 118 with abrasive alumina-coated polishing pads $(0.05 \mu \mathrm{m}$, Buehler, Whitby, ON). NEs were tested 119 for electrical conductivity and Pt size by means of cyclic voltammetry (CV). 


\section{Electrochemical Measurements}

124 Electrochemical experiments were performed using an electrochemical analyzer 125 (CHI 800B, CH Instruments, Austin, TX) with a CHI 200 Picoamp Booster (CH Instruments).

126 The current signals were recorded by the Alpha-SNOM instrument (WITec, Ulm, Germany). ${ }^{18,27}$

127 The Pt NEs were used as the SECM probe (working electrode) and a $\mathrm{Ag} / \mathrm{AgCl}$ electrode was 128 used as a combined auxiliary/reference electrode. The nanoelectrodes were biased at $-0.350 \mathrm{~V}$ 129 (vs. $\mathrm{Ag} / \mathrm{AgCl}$ ) to reduce $\mathrm{Ru}\left(\mathrm{NH}_{3}\right)_{6}{ }^{3+}$ to $\mathrm{Ru}\left(\mathrm{NH}_{3}\right)_{6}{ }^{2+}$ in a diffusion-controlled manner.

\section{SECM Experiments}

The SECM instrumentation and operation procedure has been described elsewhere in 132 great detail. ${ }^{18-19,}$ 28-29 Briefly, SECM measurements were performed on the IAME sample 133 adhered with 3M double-sided tape (conductive side exposed) onto a glass bottom petri dish 134 (P35G-0-20C, MatTek Corporation, Ashland, MA). The petri dish was mounted onto the 135 scanning stage (P-517K064, Polytec PI, Germany) of the Alpha-SNOM instrument. Utilizing 136 the Alpha-SNOM positioning system along with an inverted Nikon objective lens (50x lens, 137 N.A.: 0.55 , Japan), the SECM images were collected using the confocal mode setting within the 138 WITec software. This resulted in the real-time collection of 2D SECM images (current vs. probe 139 coordinates). The depth scan image comprised of $512 \times 200$ pixels, a scan width of $20 \mu \mathrm{m}$ and 140 depth of $10 \mu \mathrm{m}$ and an integration time of $0.01 \mathrm{~s}$ per pixel. The constant height images include 141512 x 512 pixels, a scan width and height of $20 \mu \mathrm{m}$ each and an integration time of $0.01 \mathrm{~s}$ per 142 pixel. These experiments were carried out at ambient lab conditions $\left(23 \pm 1^{\circ} \mathrm{C}\right)$.

\section{Simulation Geometry and Methodology}


The SECM imaging of the IAME sample was simulated using the finite elemental

145 analysis software, COMSOL Multiphysics v.5.3 (COMSOL, Boston, MA). The simulation

146 model was designed in full 3D geometry with a central symmetry plane. The model geometry

147 was constructed to closely represent the experimental system under study (Fig. 2a and b). The

148 SECM probe was configured to have a Pt tip size of $250 \mathrm{~nm}$ (diameter) and an RG of 10. In fact,

149 the RG of the $250 \mathrm{~nm}$ Pt NE was simplified from 30 to 10 due to minimal effects on feedback

150 current for RG values greater than $10 .^{14}$

Figure 2

153 The IAME substrate consisted of 4 sets of alternating Pt and glass microbands, each $5 \mu \mathrm{m}$ in

154 width (Fig. 2b). The microbands were designed with two differing geometries; such that the

155 simulation could be computed with flat (flush) Pt and glass microbands or raised Pt microbands

156 (110 $\mathrm{nm}$ as per manufacturer specifications) and flat glass microbands. The simplified, idealized

157 flat Pt microband structures provided a significant reduction in the complexity of the simulation

158 model. This greatly reduces the demand on system resources and computation time (nearly 10x

159 computation time with $110 \mathrm{~nm}$ step geometry). The $110 \mathrm{~nm}$ height of the Pt microbands

160 represents the true experimental geometry; however only affects the simulated curves under

161 certain conditions.

162 The simulation model was designed with a symmetry plane that passes through the center

163 of the electrode, perpendicular to the Pt microbands of the IAME sample (Fig. 2a). This allows

164 a further reduction in the complexity of the model since half of the geometry can be eliminated.

165 As a result, the number of meshing elements is halved without affecting the functionality of the 
166 model. The symmetric boundary allows the model to be mirrored across this plane and for the

167 flux of solution across the symmetry boundary into the symmetrical region opposite the 168 boundary.

The glass of the IAME sample was configured as a thin insulating boundary (Fig. 2a and

b). ${ }^{23}$ The conductive Pt microbands were set to regenerate the initial bulk solution concentration

171 of $\mathrm{Ru}\left(\mathrm{NH}_{3}\right)_{6}{ }^{3+}$, behaving as an ideal conductive boundary. The lower region of the model

172 geometry was created to facilitate the impermeable tilting substrate and has no interaction with

173 the upper region under study (Fig. 2b).

The initial solution concentration of $\mathrm{Ru}\left(\mathrm{NH}_{3}\right)_{6}{ }^{3+}$ was set at $10.0 \mathrm{mM}$ to match the stock solution concentration used in the experiments. The diffusion coefficient for $\mathrm{Ru}\left(\mathrm{NH}_{3}\right)_{6}{ }^{3+}$ was 176 taken as $6.7 \times 10^{-10} \mathrm{~m}^{2} / \mathrm{s}^{24,30-31}$ When the $250 \mathrm{~nm}$ Pt NE is biased at $-0.350 \mathrm{~V}$, the one-electron 177 reduction of $\mathrm{Ru}\left(\mathrm{NH}_{3}\right)_{6}{ }^{3+}$ to $\mathrm{Ru}\left(\mathrm{NH}_{3}\right)_{6}{ }^{2+}$ is a diffusion-controlled process that follows Fick's 178 second law of diffusion (eqn 1):

$$
\frac{\partial C_{B}}{\partial t}=D_{B}\left(\frac{\partial^{2} C_{B}}{\partial x^{2}}+\frac{\partial^{2} C_{B}}{\partial y^{2}}+\frac{\partial^{2} C_{B}}{\partial z^{2}}\right)
$$

The boundaries interfacing with the bulk solution were set to match the initial 181 concentration of $\mathrm{Ru}\left(\mathrm{NH}_{3}\right)_{6}{ }^{3+}$. This has the effect of regenerating the mediator in the region under 182 study and simulating the relatively infinite solution of the mediator in the petri dish given the 183 reaction scale which occurs at the probe tip. The probe's sheathing (quartz) boundaries were set 184 as no flux boundaries to simulate the insulating characteristics of these substrates. The Pt disk of 185 the SECM tip was configured to remove $\mathrm{Ru}\left(\mathrm{NH}_{3}\right)_{6}{ }^{3+}$ from solution, generating a concentration of $186 \quad 0 \mathrm{M}$ at the disk surface. 
A tetrahedral mesh was utilized to mesh the entire 3D simulation model (Fig. 2c). The

188 meshing element size was further refined at the regions where the greatest concentration changes

189 could be expected (electrode tip and microbands). An element scale size of $0.2 \mathrm{x}$ was set at the Pt

190 tip, while the microbands were set to $0.4 \mathrm{x}$. The minimum element size was defined as $0.01 \mu \mathrm{m}$,

191 with a maximum element growth rate of $1.25 x$. This growth rate was defined to ensure a fine

192 mesh in proximity to these boundaries of interest, up to a maximum element size of $10 \mu \mathrm{m}$.

The electrode's $x$ and $z$ positions were set as parameterized variables in the creation of

194 the model geometry. This allows the automated movement of the probe over the IAME, which

195 are simulated sequentially over numerous simulations. The sequential computation of all

196 combinations of specified parameters generates large sets of data rapidly. Concentration maps of

$197 \mathrm{Ru}\left(\mathrm{NH}_{3}\right)_{6}{ }^{3+}$ in solution were generated and the tip flux was integrated over the Pt surface (Fig. 198 2d).

Due to the symmetry plane, the integration of the semicircular electrode tip boundary has to be doubled to obtain the total electrode tip current, $i$. The surface integration follows eqn 2 :

202 where $a$ is the radius of the Pt disk, $n$ is the number of electrons transferred in the reduction of $203 \mathrm{Ru}\left(\mathrm{NH}_{3}\right)_{6}{ }^{3+}(1 \mathrm{e}), \mathrm{F}$ is the Faraday constant $(96,485 \mathrm{C} / \mathrm{mol})$, and $\mathrm{D}$ is the diffusion coefficient of $204 \mathrm{Ru}\left(\mathrm{NH}_{3}\right)_{6}{ }^{3+}$. The resulting tip current for all electrode tip locations were normalized with respect 205 to the bulk solution current at the disk electrode (simulated at a distance far away from the 206 substrate, $\left.\mathrm{i}_{\infty}\right)$, yielding:

$$
I(x, z)=\frac{i(x, z)}{i_{\infty}}
$$


208 I in this case represents the normalized current. PACs were obtained by plotting the normalized

209 tip current (eqn 3) versus the normalized distance, L (eqn 4):

$$
L=\frac{\text { tip-to-sample distance, },}{\text { electrode radius }, a}
$$

\section{Results and Discussion}

\section{SECM Imaging of an IAME by a $250 \mathrm{~nm}$ Diameter Pt NE (RG30)}

\section{Constant height mode}

217 possible. Manual polishing of these laser-pulled electrodes exposed larger Pt tip diameters than 218 those exposed by FIB. However, this exposure method is more cost-effective and the tips are 219 still significantly smaller than the commercially obtained Pt wires used in the fabrications of 220 ultramicroelectrodes (UMEs).

Figure 3

A manually polished $250 \mathrm{~nm}$ diameter Pt NE (RG 30) was used as the SECM probe to

224 electrochemically image 2 pairs of alternating $5 \mu \mathrm{m}$ glass and Pt microbands of the IAME

225 sample (Fig. 3). This electrode illustrates the effectiveness of the manually polishing laser-

226 pulled electrodes and Pt exposure method. Fig. 3a shows the 2D $(x, y)$ current map image of the 
227 microbands when the Pt NE is scanned at a constant $z$ position above the sample, where the Pt

228 microbands are represented in the bright white-yellow to orange-red colours and the glass

229 microbands by the dark blue-black colours. The image resolution collected by this $250 \mathrm{~nm} \mathrm{Pt}$

230 NE is higher than that with a micrometer diameter electrode. ${ }^{23,29,32}$ A $3 \mathrm{D}$ representation of the

$2312 \mathrm{D}$ image is shown in Fig. 3b for better visualization of the alternating nature of the microband

232 array (insulating and conductive).

From Fig. 3a, one can see that the current feedback response of the first Pt microband is

234 slightly wider than its surrounding glass microbands despite having the same physical widths(as

235 specified by the manufacturer). This increase in feedback is a result of the cumulative effects of

236 the increased signal strength of conductive substrates over insulators, the electrochemical cycling

237 between the probe and adjacent $\mathrm{Pt}$ microbands and the height difference between the $\mathrm{Pt}$ 238 microbands and interdigit glass space $(110 \mathrm{~nm})$. Therefore, a broader band of electrochemical

239 feedback will be detected as the $250 \mathrm{~nm}$ NE scans over the Pt microbands (smaller tip-to-sample 240 distance achieved). The simulation of the Pt microbands accounts for relative signal intensities

241 of the two substrates and the diffusion limited cycling of the mediator species to the defined

242 geometry. Higher feedback current was also observed as the $250 \mathrm{~nm}$ Pt NE scanned over the

243 first (left) Pt microband as opposed to the second Pt microband. This signifies that the second Pt

244 microband had a larger tip-to-sample distance as the scan progressed, indicating the IAME 245 sample or electrode are likely tilted relative to each other (Fig. 3a and b). It is also likely (due to

246 the tilt), the large RG (and therefore, diameter of the quartz sheathing) prevents the Pt tip from 247 minimizing the tip-to-sample distance further (right half of the horizontal line scans 1-3 in Fig. 248 3c-e). 
Horizontal line scans were extracted in three locations (start, middle and end of the image

251 in Fig. 3a) were overlaid onto simulated horizontal sweep curves to quantify the tip-to-sample

252 distances. Initially, the 3D IAME simulation model was simplified to have idealized flat Pt and

253 glass microbands in order to reduce the complexity of the simulation model. This height

254 differences between the Pt and glass microbands require a tip-to-glass distance to be offset by an

255 additional $0.110 \mu \mathrm{m}$.

For these experimental curves, the tip current was normalized against the steady state

257 current in the bulk. At the start of the image (line 1 (green), Fig. 3a), the $250 \mathrm{~nm}$ Pt NE scans

258 over the first glass microband which shows its insulating properties through a decrease in

259 normalized tip current $(\mathrm{I}=0.90$ (negative feedback), green circles, Fig. 3c). Following this glass

260 microband, the NE scans over the first of the two Pt microbands where the normalized tip current

261 increased to 1.08 (positive feedback). As the NE continues its horizontal scan, the adjacent

262 insulating glass microband causes another decrease in electrochemical feedback $(I=0.94)$,

263 followed by increased electrochemical feedback at the second Pt microband $(\mathrm{I}=1.04)$.

264 However, the feedback current from the second Pt microband is less than that of the first Pt

265 microband (1.04 vs 1.08), which reinforces the conclusion that either the sample or electrode is

266 tilted.

This tilt can be confirmed through the successful overlay of the experimental horizontal

268 curve onto simulated curves (Fig. 3c). A tip-to-sample distance of approximately $0.6 \mu \mathrm{m}$ was

269 reached over the first Pt and glass microbands, while the scan over the second Pt microband

270 revealed a tip-to-sample distance of 1.0 micron. This indicates a $1.53^{\circ}$ tilt across sample without

271 considering the tilting of the other plane, which is significant relative to the size of this NE.

272 Horizontal line scans taken midway (line 2, blue) and at the end of the SECM constant height 
273 image (line 3, black) also confirms that sample or electrode tilt occurs (Fig. 3d and e).

274 Therefore, for the collection of this constant height image, the $250 \mathrm{~nm}$ Pt NE was scanned 275 approximately $0.6 \mu \mathrm{m}$ above the IAME sample.

\section{Depth scan imaging}

Following the constant height image collection, the NE was raised by the $z$ piezoelectric 278 actuator of the Alpha SNOM instrument to perform SECM depth scan imaging of the 279 microbands such that the final horizontal scan of the depth scan image mirrored the $z$ position of 280 the tip in the constant height scan (Fig. 4). From the SECM depth scan, the electrochemical 281 feedback from the Pt and glass microbands can be seen as the NE approaches the IAME from 282 above. Specifically, the strong feedback current from the conductive Pt microbands onto the NE 283 is observed (minimal insulating or negative feedback from the glass microbands). As previously 284 mentioned, this is partially due to the height difference between the Pt and glass microbands (110 285 nm, equivalent to $\mathrm{L}=0.88$ ).

Figure 4

A horizontal line scan extracted near the bottom of the SECM depth scan (red line, Fig.

289 4a) revealed a tip-to-sample distance of $0.75 \mu \mathrm{m}$ when overlaid onto the simulated horizontal

290 curves (Fig. 4b). This tip-to-sample distance is comparable to the fixed $z$ position of the probe in

291 the collection of the constant height image. The immediate horizontal line scan extracted below 292 this red line indicates that the NE tip collided with the microbands (Fig. 4a). Since the RG of 
293 this NE is quite large (30), the tilt of the sample and diameter of the glass sheath prevents the Pt

294 disk from approaching the bands closer.

While achieving tip-to-sample distances of approximately $0.75 \mu \mathrm{m}$ may be ideal for

296 larger UMEs, for an electrode with an effective Pt diameter of $250 \mathrm{~nm}$, this would equal to

297 normalized distances greater than 6 for the Pt bands and 7 for the glass microbands (accounting

298 the height differences between the microbands). At these normalized distances, the fitting of

299 experimental PACs onto simulated curves would not hold much accuracy. However, the

300 normalized feedback currents from the SECM depth scan image can be used to determine their

301 corresponding tip-to-sample distances using the simulated PACs to the center of the glass and Pt

302 microbands (Fig. 5). ${ }^{1,31}$ For example, from the SECM depth scan (Fig. 4) the highest normalized

303 current towards a Pt microband was identified as 1.07. Using the simulated PACs to the center

304 of a Pt microband (red, Fig. 5), the corresponding normalized distance can be identified as 5.10.

307 Similarly, the lowest normalized current towards a glass microband was identified as 0.95 and its 308 corresponding normalized distance was identified as 6.10 (black, Fig. 5). The difference 309 between these normalized distances is therefore 1.00 or $125 \mathrm{~nm}$ (eqn 4). This height

310 discrepancy is in close agreement with manufacturer specification of $110 \mathrm{~nm}$. This also gives a 311 tilt angle of $1.43^{\circ}$ across the $x$ plane of the SECM depth scan which was similar to the tilt angle 312 of the constant height scan $\left(1.53^{\circ}\right)$. 


\section{True IAME geometry simulations with $110 \mathrm{~nm}$ as the Pt microband height}

315 Considering the height of the Pt microbands in the 3D simulations greatly increases the 316 complexity of the simulation model, requiring heavy utilization of computational resources.

317 Using experimental horizontal curves from the constant height (line 1, Fig. 3) and depth scan 318 (Fig. 4) images; these curves were overlaid onto the simulated curves with the $110 \mathrm{~nm} \mathrm{Pt}$ 319 microband height accounted for (Fig. 6). The first half of the experimental constant height curve 320 overlaid well onto the simulated curve $0.575 \mu \mathrm{m}$ above the IAME (green circles onto the solid 321 purple curve, Fig. 6a). This value agrees closely with the tip-to-sample distance determined 322 using the idealized model, estimated to be $0.6 \mu \mathrm{m}$ (Fig. 3c). When the height of the Pt 323 microbands is considered, the tip-to-sample distance reached in the depth scan image 324 corresponds to $0.725 \mu \mathrm{m}$ (red circles on solid olive curve, Fig. 6b). This value also shows close 325 agreement with the tip-to-sample distance determined from the idealized curves $(0.750 \mu \mathrm{m})$.

Using the maximum and minimum normalized currents from the SECM depth scan 329 image (Pt and glass microbands, respectively, Fig. 4), the corresponding normalized distances

330 were determined (Fig. 7). Simulated PACs were generated to the center of the Pt or glass

331 microbands, where the height difference of the microbands is accounted for in the simulation 332 model. For both normalized currents, $\left(I_{P t}=1.07\right.$ and $\left.I_{\text {glass }}=0.95\right)$, the corresponding normalized 333 distances was identified as 5.10, relative to the top of the Pt bands. This result was expected 334 since the height of the Pt microbands is considered in this simulation model. 
337 Comparison between 3D Simulation Models

338 The comparison of the results from the two different simulation models indicates that the height

339 of the Pt microbands have minimal effects on the feedback current. The height is $110 \mathrm{~nm}$,

340 which, to a $250 \mathrm{~nm}$ Pt electrode, is almost a normalized distance of 1 away $(\mathrm{L}=0.88)$. It was

341 expected with a much larger SECM probe (micron-sized), the additional surface area of the Pt

342 microband plays minimal effect, while smaller probes, such as the electrode used in these

343 experiments, would have influence on the detected feedback current. We suspect the $110 \mathrm{~nm}$

344 height difference to play a more significant role upon closer approach of the $250 \mathrm{~nm}$ Pt electrode

345 or with further reduction in the electrode size.

\section{Conclusions}

The laser-pulled quartz sealed Pt NEs were used as an SECM probe to image the Pt and

348 glass microbands of the IAME sample. SEM of these laser-pulled Pt NEs confirmed a 349 significant reduction in the diameter of the Pt wire: from $25 \mu \mathrm{m}$ to $25 \mathrm{~nm}$ (1000 times reduction).

350 However, it also revealed that the Pt wires of these NEs are commonly recessed within the pulled 351 quartz capillary. After FIB milling was used to expose the Pt tips, the resulting RG of these laser-

352 pulled NEs were found to be greater than 25. An alternative exposure method is through manual 353 (abrasive) polishing, which is simpler and more cost-effective, however the resulting NE probes 354 often have larger exposed Pt disks. Needless to say, the tip diameters of the manually polished 
355 electrodes are frequently found to be significantly smaller than the commercially obtained $\mathrm{Pt}$

356 wires (currently, the smallest commercially available is $10 \mu \mathrm{m}$ diameter (non-Wollaston)).

Here, a manually polished $250 \mathrm{~nm}$ Pt NE (RG30) was used to characterize the Pt and

358 glass microbands of an IAME sample. The submicron probe was very effective for SECM

359 imaging regardless of its high RG value. The constant height images indicated weak redox

360 cycling between $5 \mu \mathrm{m} \mathrm{Pt} \mathrm{microbands} \mathrm{which} \mathrm{were} \mathrm{separated} \mathrm{by} \mathrm{a} 5 \mu \mathrm{m}$ glass interdigit space

361 (microband). In conjunction with theoretical simulations completed in COMSOL Multiphysics,

362 the tip-to-sample distances could only be determined through fitting of the experimental

363 horizontal line scans. Utilizing an idealized IAME geometry (flat microbands), it was

364 determined that the $250 \mathrm{~nm}$ Pt NE was scanned approximately $0.6 \mu \mathrm{m}$ above the IAME sample.

365 In addition, the presence of either sample or electrode tilt of $1.53^{\circ}$ was identified in the 366 electrodes imaging of the IAME.

The SECM depth scan image obtained over the IAME demonstrated strong 368 electrochemical feedback upon approach to the conductive bands. This was due to the smaller 369 normalized distances achieved to the Pt microbands since there is a height discrepancy $(110 \mathrm{~nm})$ 370 between the Pt and glass microbands. The final horizontal line scan of the SECM probe in the 371 experimental depth scan was set to mirror the fixed $z$ position of the probe during the collection 372 of the constant height image. Utilizing a horizontal line scan at the bottom of the depth scan 373 image revealed a tip-to-sample distance in close agreement to that from the constant height 374 image $(0.75 \mu \mathrm{m})$. Since this tip-to-sample distance corresponds to a normalized distance greater 375 than 6, the fitting of experimental PACs to simulated curves becomes difficult. Therefore, the 376 maximum and minimum normalized currents were matched to the simulated PACs to the 377 respective Pt and glass microbands. This allowed the determination of the corresponding 
378 normalized tip-to-substrate distances to the Pt and glass microbands. Using the idealized flat

379 geometry of the bands the normalized distances revealed a $125 \mathrm{~nm}$ height variation which is

380 similar to manufacturer's specifications $(110 \mathrm{~nm})$. At these tip-to-sample distances, the height of

381 the Pt microbands was found to play a minimal effect on the feedback current. 3D simulation

382 modelling was utilized for the first time, allowing characterization of these horizontal curves

383 above and across the microbands of the IAME to quantify the probe's tip-to-sample distance and

384 sample characteristics, such as tilt and height of the microbands.

385 Due to the size of the SECM probe, it was expected that the height of the Pt microbands

386 would play a significant role on the feedback current detected at the IAME. However, finite

387 elemental analysis simulations of this SECM study accounting for the height of the Pt

388 microbands revealed minimal effect on current feedback. These results indicate that the

389 idealized geometry of the IAME (flat microbands) can be used in the simulations, greatly

390 reducing the computational times and resources required. The combinations of these 3D finite

391 elemental analysis stimulations and nanoelectrodes will be advantageous in nanoscale SECM

392 studies.

393

394

395 Acknowledgements

396 We thank the Natural Sciences and Engineering Research Council of Canada (NSERC, DG

397 RGPIN-2013-201697 and SPG STPGP-2016-493924), Canada Foundation for

398 Innovation/Ontario Innovation Trust (CFI/OIT, 9040), Premier's Research Excellence Award 
399 (PREA, 2003) for financial support of this work. ZD acknowledges The University of Western 400 Ontario and the Faculty of Science for a Distinguished Research Professorship (2014-2015) and 401 Faculty Scholar Award (2015-2016). MSML and FPF acknowledge the assistance of an Ontario 402 Graduate Scholarship (2015-2017 and 2017-2018). We are grateful to Dr. Todd S. Simpson from 403 the Western Nanofabrication Lab for his assistance in FIB-milling and SEM imaging and the 404 Mauzeroll group for the help of nanoelectrode fabrication.

405

\section{References}

407 1. Bard, A. J.; Fan, F. R. F.; Kwak, J.; Lev, O., Anal. Chem. 1989, 61, 132. doi: 408 10.1021/ac00177a011.

409 2. Kwak, J.; Bard, A., Anal. Chem. 1989, 61, 1221 doi: 10.1021/ac00186a009.

$410 \quad 3 . \quad$ Engstrom, R. C.; Weber, M.; Wunder, D. J.; Burgess, R.; Winquist, S., Anal. Chem. 411 1986, 58, 844. doi: 10.1021/ac00295a044.

$412 \quad 4 . \quad$ Engstrom, R. C.; Meaney, T.; Tople, R.; Wightman, R. M., Anal. Chem. 1987, 59, 2005. 413 doi: 10.1021/ac00142a024.

414 5. Eifert, A.; Mizaikoff, B.; Kranz, C., Micron 2015, 68, 27. doi: 415 10.1016/j.micron.2014.08.008.

$416 \quad$ 6. Ufheil, J.; Hess, C.; Borgwarth, K.; Heinze, J., Phys. Chem. Chem. Phys. 2005, 7, 3185. 417 doi: 10.1039/b505189d.

418 7. Barker, A. L.; Macpherson, J. V.; Slevin, C. J.; Unwin, P. R., J. Phys. Chem. B 1998, 419 102, 1586. doi: 10.1021/jp973370r.

420 8. Nioradze, N.; Kim, J.; Amemiya, S., Anal. Chem. 2010, 83, 828. doi: 421 10.1021/ac102352v.

422 9. Li, M. S. M.; Filice, F. P.; Henderson, J. D.; Ding, Z. F., J. Phys. Chem. C 2016, 120, 423 6094. doi: 10.1021/acs.jpcc.6b00453.

424 10. Bard, A. J.; Li, X.; Zhan, W., Biosens. Bioelectron. 2006, 22, 461. doi: 425 10.1016/j.bios.2006.04.028. 
426 11. Wittstock, G.; Burchardt, M.; Pust, S. E.; Shen, Y.; Zhao, C., Angew. Chem. Int. Ed. 427 2007, 46, 1584. doi: 10.1002/anie.200602750.

428 12. Liu, B.; Cheng, W.; Rotenberg, S. A.; Mirkin, M. V., J. Electroanal. Chem. 2001, 500, 429 590. doi: 10.1016/S0022-0728(00)00436-8.

430 13. Li, X.; Bard, A. J., J. Electroanal. Chem. 2009, 628, 35. doi: 431 10.1016/j.jelechem.2009.01.002.

432 14. Bard, A. J.; Mirkin, M. V. Scanning Electrochemical Microscopy. 2 ed.; CRC Press2012; 433 p 670.

434 15. Takahashi, Y.; Kumatani, A.; Shiku, H.; Matsue, T., Anal. Chem. 2017, 89, 342. doi: 435 10.1021/acs.analchem.6b04355.

436 16. Tian, H.; Li, Y.; Shao, H.; Yu, H. Z., Anal Chim Acta 2015, 855, 1. doi: $437 \quad$ 10.1016/j.aca.2014.06.030.

438 17. She, Z.; Topping, K.; Dong, B.; Shamsi, M. H.; Kraatz, H.-B., Chem. Commun. 439 (Cambridge, U. K.) 2017, 53, 2946. doi: 10.1039/C7CC00863E.

440 18. Zhang, M. M. N.; Long, Y. T.; Ding, Z., Chem. Cent. J. 2012, 6, 20. doi: 10.1186/1752441 153X-6-20.

442 19. Li, M. S. M.; Filice, F. P.; Ding, Z., J. Inorg. Biochem. 2014, 136, 177. doi: 443 10.1016/j.jinorgbio.2014.02.009.

444 20. Filice, F. P.; Li, M. S. M.; Henderson, J. D.; Ding, Z., Anal. Chim. Acta 2016, $908,85$. 445 doi: 10.1016/j.aca.2015.12.027.

446 21. Mezour, M. A.; Morin, M.; Mauzeroll, J., Anal. Chem. 2011, 83, 2378. doi: 447 10.1021/ac102482f.

$448 \quad 22 . \quad$ Danis, L.; Gateman, S. M.; Snowden, M. E.; Halalay, I. C.; Howe, J. Y.; Mauzeroll, J., Electrochim. Acta 2015, 162, 169. doi: 10.1016/j.electacta.2014.10.027.

450 23. Li, M. S. M.; Filice, F. P.; Ding, Z. F., J Electroanal Chem 2016, 781, 126. doi: 451 10.1016/j.jelechem.2016.06.043.

452 24. Guo, J.; Amemiya, S., Analytical Chemistry 2005, 77, 2147.

453 25. Danis, L.; Snowden, M. E.; Tefashe, U. M.; Heinemann, C. N.; Mauzeroll, J., 454 Electrochim Acta 2014, 136, 121. doi: 10.1016/j.electacta.2014.05.047.

455 26. Danis, L.; Polcari, D.; Kwan, A.; Gateman, S. M.; Mauzeroll, J., Anal. Chem. 2015, 87, 456 2565. doi: 10.1021/ac503767n.

457 27. Zhang, M. M. N.; Long, Y.-T.; Ding, Z., Journal of Inorganic Biochemistry 2012, 108, 458 115. doi: http://dx.doi.org/10.1016/j.jinorgbio.2011.11.010. 
459 28. Zhang, M.-N.; Ding, Z.; Long, Y.-T., Analyst 2015. doi: 10.1039/C5AN01148E.

460 29. Filice, F. P.; Li, M. S. M.; Henderson, J. D.; Ding, Z., J. Phys. Chem. C 2015, 119, 461 21473. doi: 10.1021/acs.jpcc.5b05568.

462 30. Miao, W.; Ding, Z.; Bard, A. J., Journal of Physical Chemistry B 2002, 106, 1392.

463 31. Bard, A. J.; Faulkner, L. R. Electrochemical Methods: Fundamentals and Applications, 464 2nd Edition. 2 ed.; John Wiley \& Sons, Inc.2001; p 864.

465 32. Zhu, R.; Ding, Z., Can. J. Chem. 2005, 83, 1779. doi: 10.1139/v05-189.

\section{Figure Captions}

Fig. 1. Labelled SEM images of a laser-pulled Pt nanoelectrode (a) before and (b) after FIB-

469 milling to expose the Pt tip. The Pt wire was reduced by a factor of one thousand ( $25 \mu \mathrm{m}$ to 25

$470 \mathrm{~nm}$ ) and an RG greater than 25 can be observed.

471 Fig. 2. (a) The labelled stimulated model geometry of the SECM analysis of an IAME sample

472 with the symmetry plane shown in blue. (b) A zoomed in and labelled model of the Pt and glass

473 microbands, where the heights of the Pt microbands are taken into account $(110 \mathrm{~nm})$. (c) The

474 tetrahedrally-meshed simulation model with further refinement of the mesh at the electrode tip 475 and the microbands of the IAME to improve the accuracy of the simulation. (d) An example of

476 a simulated concentration map of the experiment, where the SECM probe is positioned in bulk

477 solution (several tip radii distances above the sample).

478 Fig. 3. (a) and (b) are the constant height SECM images of two alternating pairs of Pt and glass

479 microbands collected in $10 \mathrm{mM} \mathrm{Ru}\left(\mathrm{NH}_{3}\right)_{6}{ }^{3+}$ solution with $0.1 \mathrm{M} \mathrm{KCl}$ as the supporting 480 electrolyte. (a) is the 2D current map view and (b) is a 3D view to provide stronger visualization 481 of the electrochemical behaviour of the Pt and glass microbands. The scale bars in the images 
482 represent $4 \mu \mathrm{m}$ and the current scale bar in (a) is been normalized against the steady state 483 current. (c)-(e) are the experimental horizontal line scans taken at the locations specified in (a) 484 and have been overlaid over simulated curves generated at $0.50,0.75,1.00,2.00$ and $3.00 \mu \mathrm{m}$ 485 above the sample. From successful overlays, the $250 \mathrm{~nm}$ Pt NE was scanned approximately 0.6 $486 \mu \mathrm{m}$ above the sample. The simulation geometry of the working electrode was simplified to a 250 487 nm Pt NE with an RG of 10.

488 Fig. 4. (a) SECM depth scan image towards the glass and Pt microbands of the IAME sample. A 489 horizontal line scan (red pentagons) is extracted near the end of the scan. (b) The experimental 490 horizontal scan is overlaid onto theoretical curves to quantify tip-to-sample distance.

491 Fig. 5. The simulated PACs towards the center of (flat) Pt and glass microbands (red and black, 492 respectively). The normalized currents from the SECM depth scan were used to identify their 493 corresponding normalized distance.

494 Fig. 6. Horizontal line scans extracted from the (a) constant height image (line scan 1) and (b) 495 depth scan image overlaid onto theoretical curves generated over an IAME with the height of the 496 Pt microbands $(110 \mathrm{~nm})$ taken into account.

497 Fig. 7. Theoretical PACs of a $250 \mathrm{~nm}$ Pt NE's approach to $110 \mathrm{~nm} \mathrm{Pt}$ and glass microbands (red 498 and black) of the IAME. 

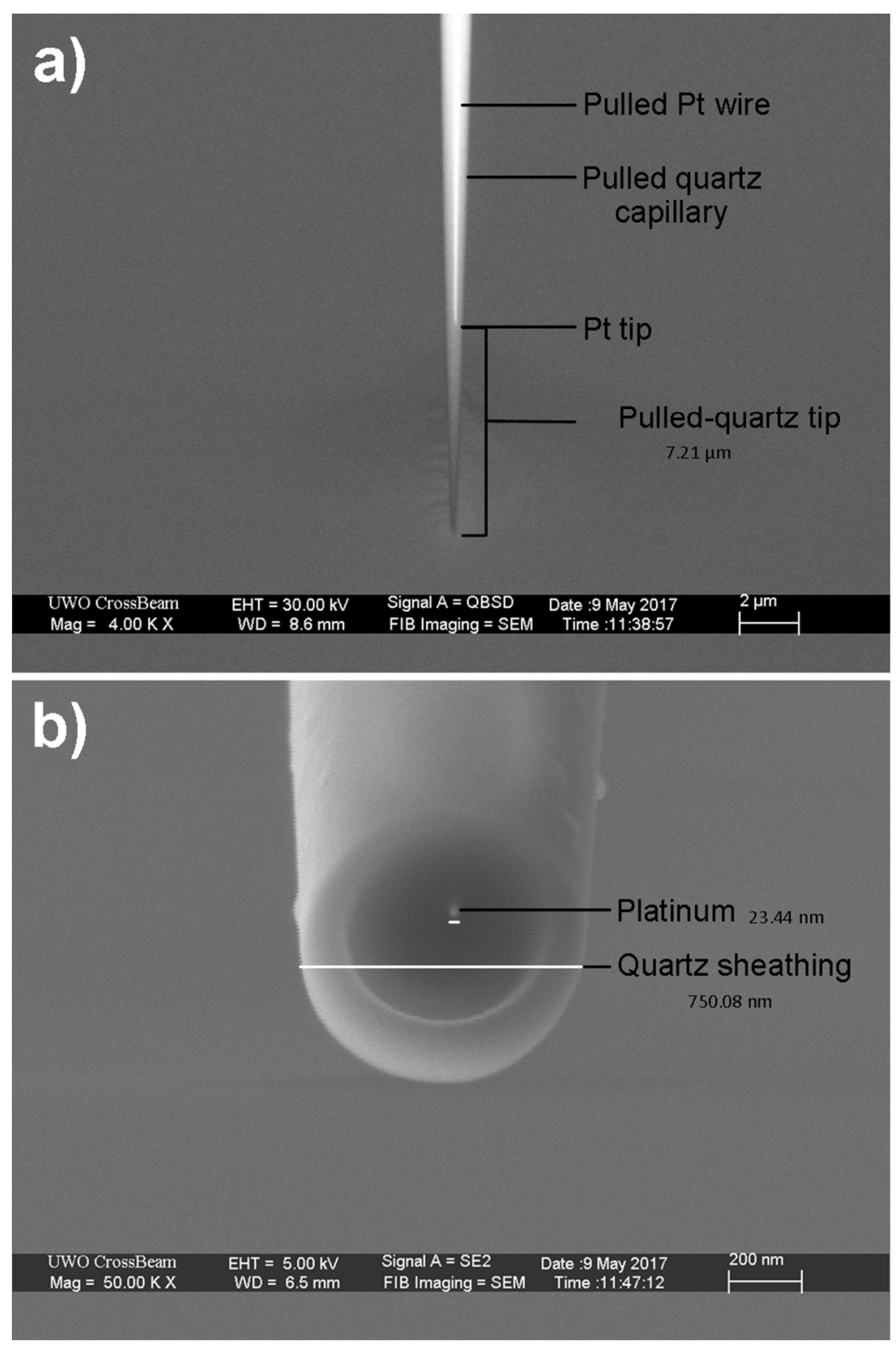

Fig. 1. Labelled SEM images of a laser-pulled Pt nanoelectrode (a) before and (b) after FIB-milling to expose the Pt tip. The Pt wire was reduced by a factor of one thousand ( $25 \mu \mathrm{m}$ to $25 \mathrm{~nm}$ ) and an RG greater than 25 can be observed.!! +

$82 \times 121 \mathrm{~mm}(299 \times 299$ DPI $)$ 


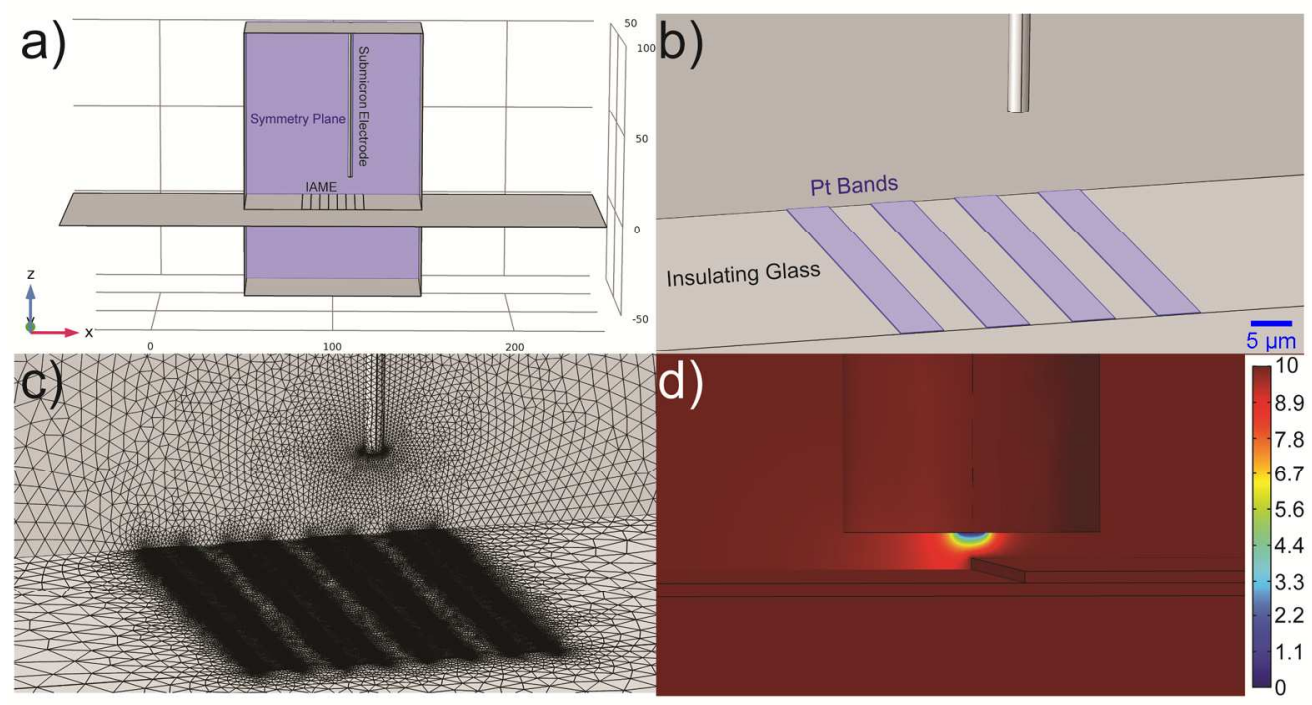

Fig. 2. (a) The labelled stimulated model geometry of the SECM analysis of an IAME sample with the symmetry plane shown in blue. (b) A zoomed in and labelled model of the Pt and glass microbands, where the heights of the Pt microbands are taken into account $(110 \mathrm{~nm})$. (c) The tetrahedrally-meshed simulation model with further refinement of the mesh at the electrode tip and the microbands of the IAME to improve the accuracy of the simulation. (d) An example of a simulated concentration map of the experiment, where the SECM probe is positioned in bulk solution (several tip radii distances above the sample).

$186 \times 102 \mathrm{~mm}(299 \times 299 \mathrm{DPI})$ 
a)
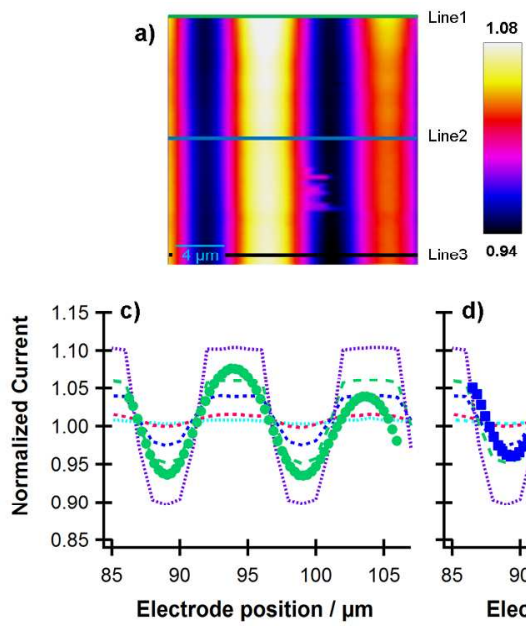
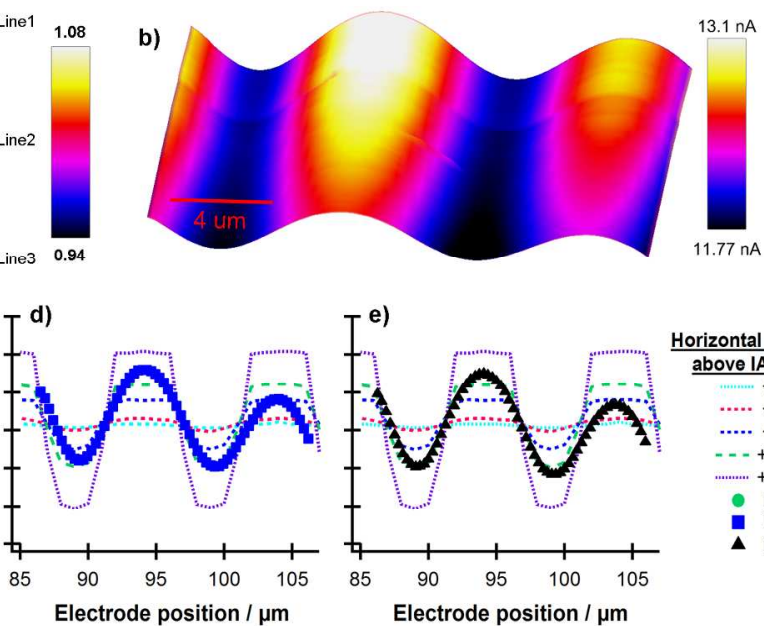

orizontal line scans above IAME / $\mu \mathrm{m}$ $+3.00$ -... +2.00 $\cdots+1.00$ $-++0.750$ -...+ +0.500 - Line 1 - Line 2 - Line 3

Fig. 3. (a) and (b) are the constant height SECM images of two alternating pairs of Pt and glass microbands collected in $10 \mathrm{mM} \mathrm{Ru}(\mathrm{NH} 3) 63+$ solution with $0.1 \mathrm{M} \mathrm{KCl}$ as the supporting electrolyte. (a) is the 2D current map view and (b) is a $3 \mathrm{D}$ view to provide stronger visualization of the electrochemical behaviour of the Pt and glass microbands. The scale bars in the images represent $4 \mu \mathrm{m}$ and the current scale bar in (a) is been normalized against the steady state current. (c)-(e) are the experimental horizontal line scans taken at the locations specified in (a) and have been overlaid over simulated curves generated at $0.50,0.75,1.00,2.00$ and $3.00 \mu \mathrm{m}$ above the sample. From successful overlays, the $250 \mathrm{~nm}$ Pt NE was scanned approximately $0.6 \mu \mathrm{m}$ above the sample. The simulation geometry of the working electrode was simplified to a $250 \mathrm{~nm}$ Pt NE with an RG of 10.!! +

$194 \times 94 \mathrm{~mm}(299 \times 299$ DPI $)$ 


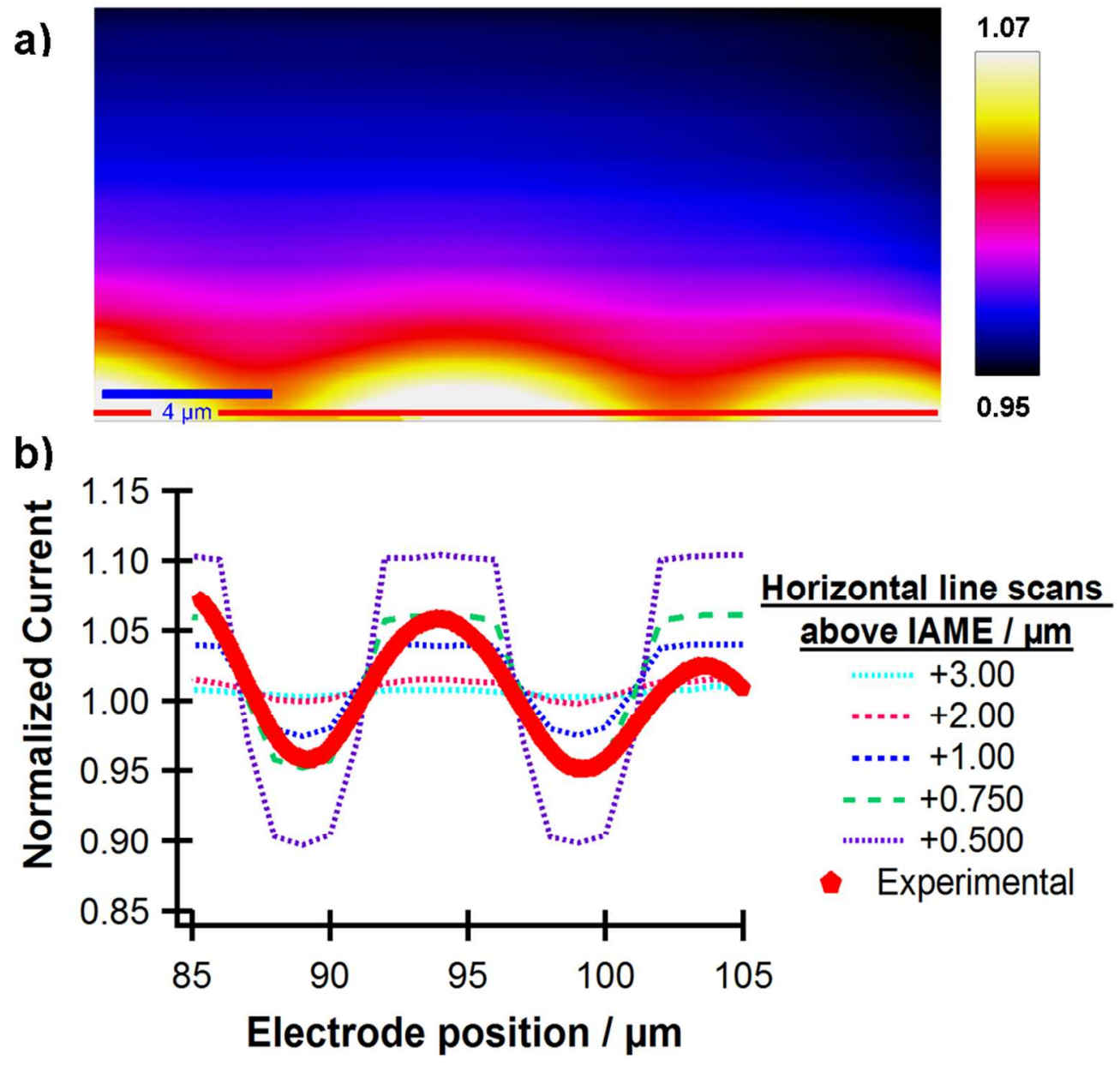

Fig. 4. (a) SECM depth scan image towards the glass and Pt microbands of the IAME sample. A horizontal line scan (red pentagons) is extracted near the end of the scan. (b) The experimental horizontal scan is overlaid onto theoretical curves to quantify tip-to-sample distance.!! +

$$
98 \times 94 \mathrm{~mm}(299 \times 299 \mathrm{DPI})
$$




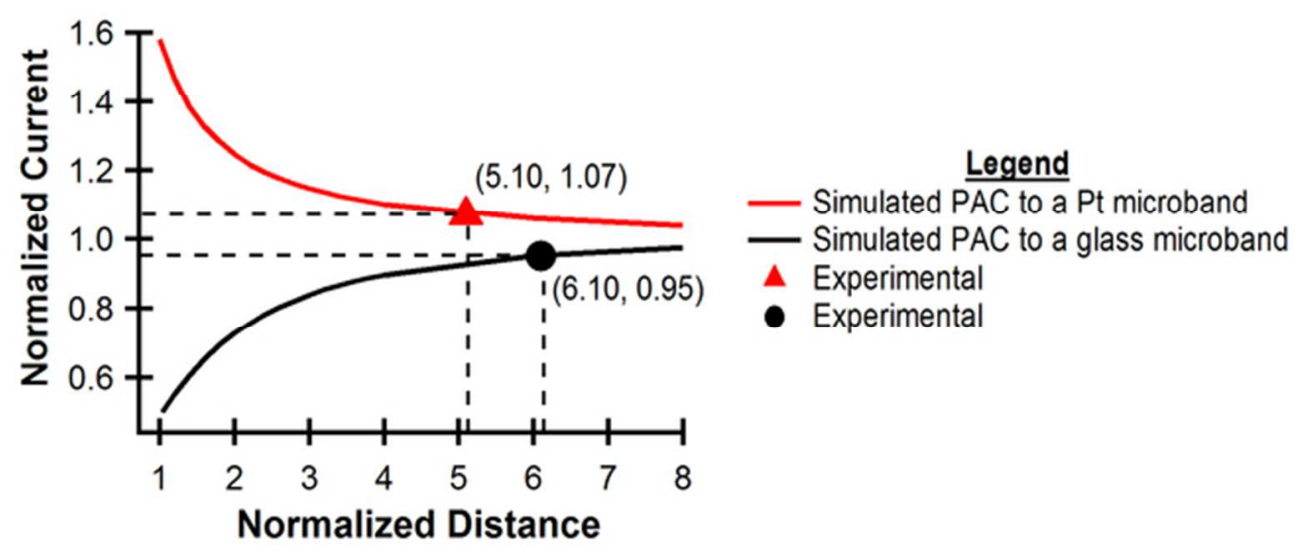

Fig. 5. The simulated PACs towards the center of (flat) Pt and glass microbands (red and black, respectively). The normalized currents from the SECM depth scan were used to identify their corresponding normalized distance.

$56 \times 25 \mathrm{~mm}(300 \times 300$ DPI $)$ 

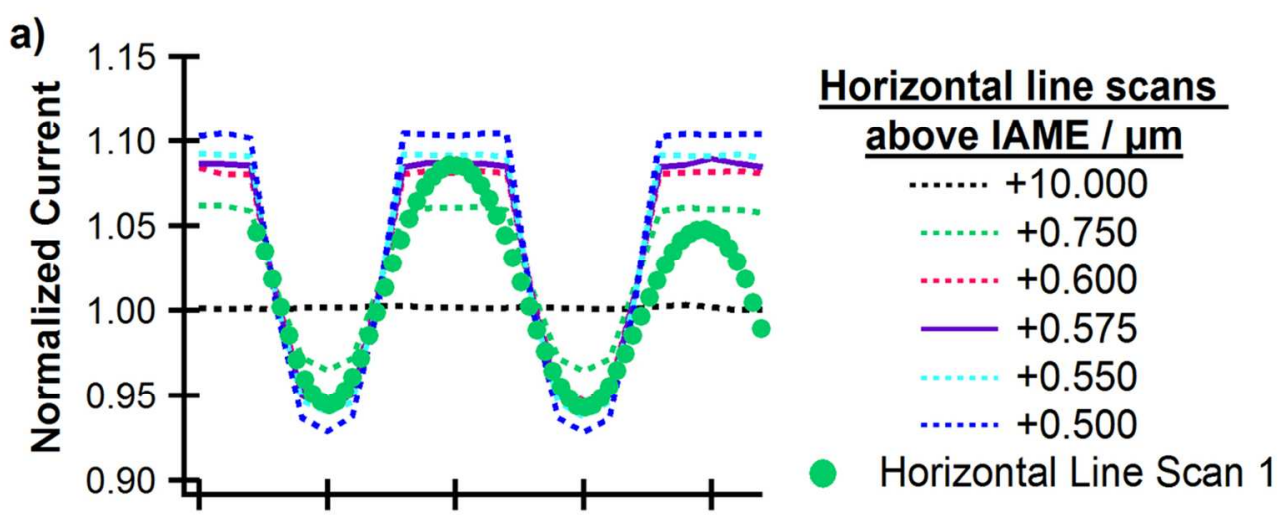

b)

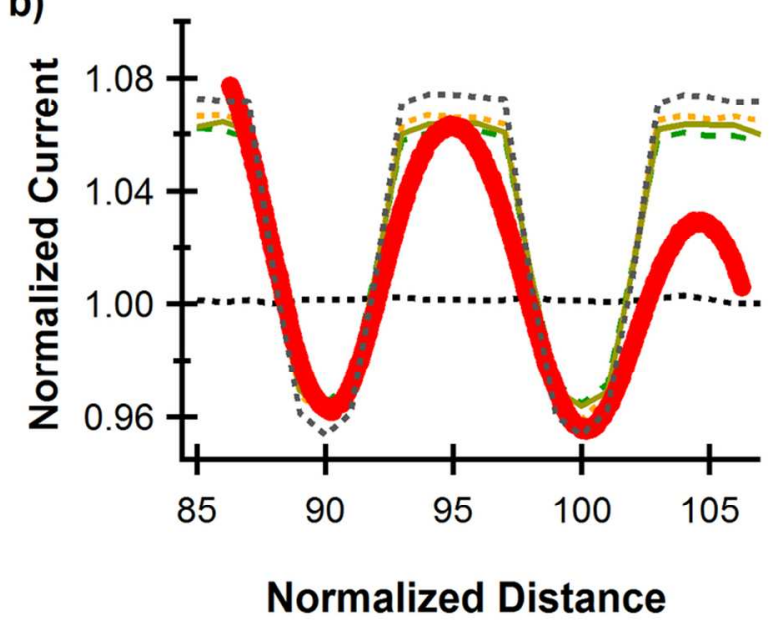

Horizontal line scans above IAME / $\mu \mathrm{m}$

$\cdots \cdot \cdots+10.000$

$--\cdot+0.750$

$+0.725$

$+0.700$

$+0.650$

- Depth Scan Line 1

Fig. 6. Horizontal line scans extracted from the (a) constant height image (line scan 1) and (b) depth scan image overlaid onto theoretical curves generated over an IAME with the height of the Pt microbands (110 $\mathrm{nm}$ ) taken into account.

$104 \times 96 \mathrm{~mm}(300 \times 300$ DPI $)$ 


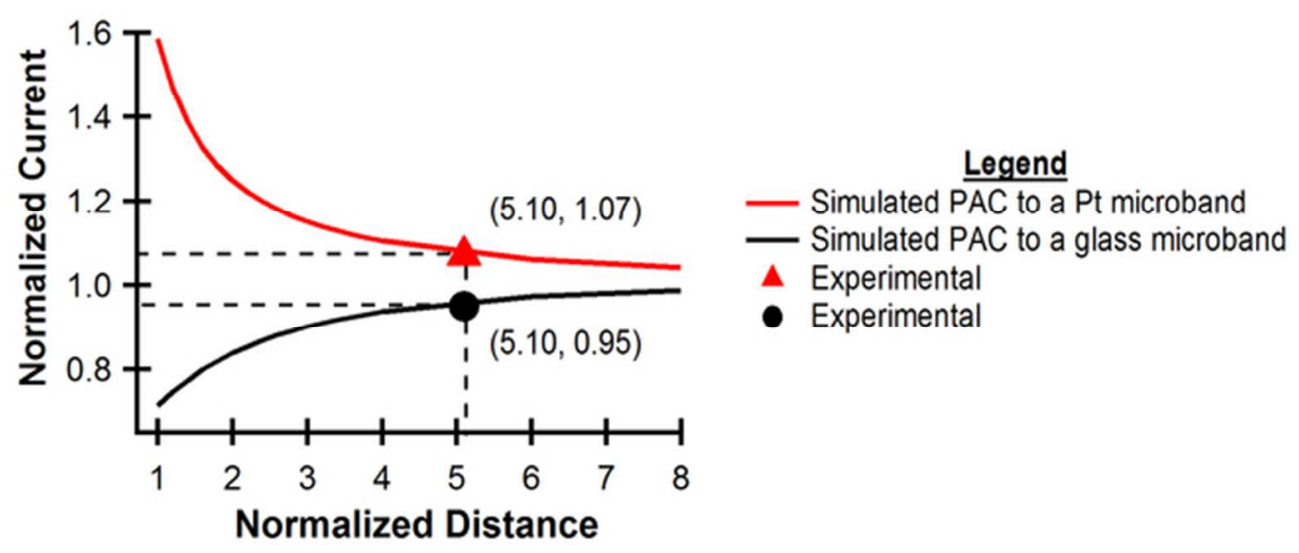

Fig. 7. Theoretical PACs of a $250 \mathrm{~nm}$ Pt NE's approach to $110 \mathrm{~nm}$ Pt and glass microbands (red and black) of the IAME.

$56 \times 25 \mathrm{~mm}(300 \times 300 \mathrm{DPI})$ 